\title{
Etnoconhecimento de pescadores artesanais na comunidade Bebedouro, Santo
}

\author{
Amaro, Brasil \\ Ethnoknowledge of artisanal fishermen in the Bebedouro community, Santo Amaro, Brazil \\ Etnoconocimiento de los pescadores artesanales de la comunidad Bebedouro, Santo Amaro, Brasil
}

Recebido: 25/06/2021 | Revisado: 04/07/2021 | Aceito: 07/07/2021 | Publicado: 17/07/2021

\author{
Ádila Patrícia Chaves Silva \\ ORCID: https://orcid.org/0000-0003-0166-1057 \\ Universidade Estadual do Maranhão, Brasil \\ E-mail: adilachaves@gmail.com \\ Nelson Mateus Silva Costa \\ ORCID: https://orcid.org/0000-0002-8877-6166 \\ Universidade Estadual do Maranhão, Brasil \\ E-mail: cmateus055@gmail.com \\ Manoel Cleber Sampaio Silva \\ ORCID: https://orcid.org/0000-0003-4942-0500 \\ Universidade Estadual do Maranhão, Brasil \\ E-mail: manoel.silva@ifma.edu.br \\ Ricardo Pinto dos Santos \\ ORCID: https://orcid.org/0000-0001-5461-345X \\ Universidade Estadual do Maranhão, Brasil \\ E-mail: ricardo13.rpds@gmail.com \\ Ione de Oliveira Gomes \\ ORCID: https://orcid.org/0000-0002-1047-9006 \\ Universidade Estadual do Maranhão, Brasil \\ E-mail: ionedeoliveiragomes@gmail.com \\ Janderson Bruzaca Gomes \\ ORCID: https://orcid.org/0000-0003-3356-4112 \\ Universidade Estadual do Maranhão, Brasil \\ E-mail: Jandersonbruzaca@ hotmail.com \\ Zafira da Silva de Almeida \\ ORCID: https://orcid.org/0000-0002-8295-5040 \\ Universidade Estadual do Maranhão, Brasil \\ E-mail: zafiraalmeida@hotmail.com
}

\begin{abstract}
Resumo
O presente trabalho objetivou analisar o conhecimento etnoictiológico dos pescadores da comunidade de Bebedouro, pertencente ao município de Santo Amaro, Maranhão. Através de questionários semiestruturados foi possível observar que os pescadores desenvolvem apenas a pesca artesanal como fonte de economia. Tanto os homens e as mulheres exercem a profissão, sendo que essa atividade começa desde a infância. $\mathrm{Na}$ análise dos questionários constataram 14 espécies de peixes. Para a maioria dos pescadores, todos os peixes são diferenciados entre peixes adultos e filhotes pelo seu tamanho. Em relação a distribuição espacial das etnoespécies, os pescadores diferenciam os ambientes de acordo com os locais onde estes são capturados e as formas utilizadas para captura, expressando com isso a posição que os peixes ocupam na coluna d'água. Os pescadores associam a época de reprodução com o comportamento de desova. Os pescadores de Bebedouro desconhecem o período defeso, afirmam não receber o seguro defeso, dessa forma, não existe um período em que não há pesca. A comunidade de Bebedouro possui um potencial em biodiversidade e a pesca é essencial para a comunidade, podemos caracterizar o etnoconhecimento como uma importante ferramenta, considerando a falta de estudo e assistência para o efetivo desenvolvimento de suas atividades, restando apenas os conhecimentos adquiridos através das gerações e que os auxiliam na pesca e conservação dos recursos explorados.
\end{abstract}

Palavras-chave: Conhecimento tradicional; Pesca; Recursos pesqueiros.

\section{Abstract}

This study aimed to analyze the ethnoichthyological knowledge of fishermen in the community of Bebedouro, belonging to the district of Santo Amaro, Maranhão. Through semi-structured questionnaires it was possible to observe that fishermen only develop handmade fishing as a source of savings. Both men and women exercise the profession, and this activity begins since the childhood. In the analysis of the questionnaires, 14 species of fish were found. For most fishermen, all fish are differentiated between adult and young fish by their size. Regarding the spatial 
distribution of ethnospecies, fishermen differentiate the environments according to the places where they are caught and the forms used for capture, expressing this way the position that the fish occupy in the water column. Fishermen associate the breeding time with the spawning behavior. Bebedouro fishermen are unaware of the closed season, claim not to receive closed-end insurance, thus, there is no period when fishing is forbidden. The community of Bebedouro has a potential in biodiversity and fishing is essential for the community, we can characterize ethnoknowledge as an important tool, considering the lack of study and assistance for the effective development of its activities, leaving only the knowledge acquired through generations and that assist them in the fishing practice and conserving exploited resources.

Keywords: Traditional knowledge; Fishing; Fishing resource.

\section{Resumen}

Este estudio tuvo como objetivo analizar el conocimiento etnoictiológico de los pescadores de la comunidad de Bebedouro, perteneciente al municipio de Santo Amaro, Maranhão. A través de cuestionarios semiestructurados se pudo observar que los pescadores solo tienem la pesca artesanal como labora. Hombres, así como mujeres, ejercen la profesión, y esta actividad comienza desde la niñez. En el análisis de los cuestionarios se encontraron 14 especies de peces. Para la mayoría de los pescadores, todos los peces se diferencian entre adultos y jóvenes por su tamaño. En cuanto a la distribución espacial de las etnoespecies, los pescadores diferencian los ambientes según los lugares donde son capturados y las formas que utilizan para capturarlos, expresando así la posición que ocupan los peces en la columna de agua. Los pescadores asocian la temporada de reproducción con el comportamiento de desove. Los pescadores de Bebedouro desconocen la veda, afirman no recibir un seguro de duración determinada, por lo que no hay período en el que no haya pesca. La comunidad de Bebedouro tiene potencial en biodiversidad y la pesca es fundamental para la comunidad, podemos caracterizar el etnoconocimiento como una herramienta importante, considerando la falta de estudio y asistencia para el desarrollo efectivo de sus actividades, dejando solo los conocimientos adquiridos a través de generaciones y que les ayuden en la pesca y conservación de los recursos explotados.

Palabras clave: Conocimiento tradicional; pescar; Recursos pesqueiros.

\section{Introdução}

A pesca é uma atividade de grande relevância econômica por representar uma importante fonte de alimento e renda para uma representativa parcela da população mundial (FAO, 2016). Sobretudo, a pesca artesanal tem uma importância social, local e regional, e por tradição se tornou uma fonte de subsistência para populações ribeirinhas, que dependem da pesca e das atividades com ela relacionadas (Santos et al., 2012).

A pesca proporciona aos pescadores um conhecimento empírico não somente das estratégias de pesca, mas sobre a fauna aquática, como as espécies-alvos da captura ou mesmo de outras espécies presentes nos pesqueiros (Brito et al., 2015). Os pescadores exploram o ambiente aquático de forma peculiar e adquirem conhecimentos sobre a natureza, além de estabelecer uma grande diversidade de interações com o ambiente (Ramires \& Molina, 2004).

O etnoconhecimento é o saber que as populações humanas possuem acerca do mundo ao nosso redor, principalmente sobre os recursos naturais disponíveis no ambiente (Diegues, 2000). Dentro desse etnoconhecimento se destaca a etnoictiologia que investiga a interação entre pescadores e os peixes sob os mais diversos aspectos, incluindo táticas de pesca, migração, reprodução, dieta e atribuição de nomes aos peixes (etnotaxonomia) (Begossi et al., 2002).

E é com a valorização destes conhecimentos e das práticas desenvolvidas por estas populações que se pode vislumbrar ações de uso e conservação dos recursos mais conectadas e coerentes com a realidade local (Da Cunha, 2011). Portanto, este trabalho teve como objetivo principal analisar o conhecimento etnoictiológico dos pescadores da comunidade de Bebedouro, pertencente ao município de Santo Amaro.

\section{Metodologia}

\subsection{Caracterização da área}

O município de Santo Amaro está situado no limite oeste do Parque Nacional dos Lençóis Maranhenses - PNLM. O Parque apresenta clima tropical caracterizado por temperatura média superior a $18^{\circ} \mathrm{C}$ e um regime pluviométrico que define 
uma estação chuvosa (janeiro a junho) e outra seca (julho a dezembro). Possui uma vegetação de restinga e a presença de manguezais isolados (IBAMA, 2004).

A comunidade de Bebedouro, localizada em Santo Amaro, está compreendida entre as coordenadas de $2^{\circ} 28^{\prime} 39.2^{\prime \prime} \mathrm{S}$ e $43^{\circ} 15^{\prime} 57.6^{\prime}$ W. A pesca nessa comunidade é desenvolvida principalmente no Lago de Santo Amaro, caracterizado por apresentar bancos arenosos, possuir margens lacustres que em determinados trechos, em virtude da pequena declividade, ficam sujeitos a inundações no período chuvoso (IBAMA, 2004).

\subsection{Coleta dos dados}

Para esta pesquisa foi utilizada questionários semiestruturados com questões abertas e fechadas, para permitir ampla coleta de informações, contendo perguntas relacionadas ao etnoconhecimento da ictiofauna da comunidade. Esse tipo de metodologia permite a captação imediata e corrente da informação desejada (Ludke \& André, 1986).

Os pescadores foram selecionados a partir da técnica "Bola de Neve" (Monteles, 2010) que consiste em um processo de coleta de informações, que procura tirar proveito das redes sociais dos entrevistados identificados para fornecer ao pesquisador um conjunto cada vez maior de contatos potenciais, sendo que o processo pode ser finalizado a partir do critério de saturação.

As etnoespécies foram nomeadas pelos pescadores e para a identificação taxonômica usou-se como base as referências de Piorski et al., (2017). Foram totalizados 20 entrevistados e as respostas foram cedidas de forma voluntária e por meio de consentimento prévio. Os dados obtidos foram tabulados em planilhas do Microsoft Excel (2013), a partir dos quais foram construídos gráficos e tabelas para melhor visualização dos resultados. A análise foi realizada comparando os resultados obtidos com aqueles já presentes na literatura científica.

\section{Resultados e Discussão}

Os pescadores residentes da comunidade de Bebedouro desenvolvem apenas a pesca artesanal como fonte de economia. Valores, tradições e percepções permanecem na cultura desses pescadores e exercem um papel fundamental no cotidiano dessa comunidade, principalmente na maneira como a pesca é desenvolvida, o que caracteriza a forma como os pescadores manejam os estoques pesqueiros, dados inclusive pelo tempo de pesca, que variou de 10 anos a 48 anos. Além do mais, tanto os homens e as mulheres exercem a profissão, sendo que essa atividade começa desde a infância.

\subsection{As etnoespécies}

$\mathrm{Na}$ análise dos questionários constataram 14 espécies de peixes, observou-se que além do profundo conhecimento dos pescadores, o grupo de entrevistados utilizam uma grande variedade de peixes para sua alimentação e para comercialização (Tabela 1). Resultado semelhante foi relatado por Massena et al., (2014), em que foi encontrado uma grande abundância de peixes na Vila Cachoeira, Ilhéus, Bahia, tais como a Hoplias Malabaricus, Astyanax sp., Oreochromis sp. 
Tabela 2. Principais espécies pescadas em Bebedouro, Santo Amaro, Maranhão.

\begin{tabular}{|c|c|c|}
\hline Nome vulgar & Nomeclatura cientifica & Utilização \\
\hline Cabeça-seca & Hoplerythrinus unitaeniatus & Alimentação e comércio \\
\hline Cangati & Trachelyopterus galeatus & Alimentação e comércio \\
\hline Cará-bicudo & Satanoperca jurupari & Alimentação e comércio \\
\hline Cará-roxo & Cichlasoma cf. zarskei & Alimentação e comércio \\
\hline Cascudo & Hypostomus sp. & Alimentação e comércio \\
\hline Corvina & Plagioscion squamosissimus & Alimentação e comércio \\
\hline Pataca & Metynnis cf. lippincottianus & Alimentação e comércio \\
\hline Piaba & Astyanax sp. & Alimentação e comércio \\
\hline Piau & Leporinus cf. friderici & Alimentação e comércio \\
\hline Piranha-branca & Serrasalmus rhombeus & Alimentação e comércio \\
\hline Sarapó & Gymnotus carapo & Alimentação e comércio \\
\hline Tambaqui & Colossoma macropomum & Alimentação e comércio \\
\hline Tilápia & Oreochromis sp. & Alimentação e comércio \\
\hline Traíra & Hoplias malabaricus & Alimentação e comércio \\
\hline
\end{tabular}

Fontes: Autores.

A traíra (Hoplias malabaricus), o cará roxo (Cichlasoma cf. zarskei) e a tilápia (Oreochromis sp.), são os mais mencionados, pois representam para a comunidade as espécies de maior valor comercial e demanda de clientes. É importante mencionar que a tilápia não era encontrada na região, apesar de ser uma espécie exótica, é amplamente cultivada no Brasil, comum na região, segundo os pescadores, a tilápia apareceu após o rompimento de uma barragem, porém, os pescadores adotaram essa espécie, pois encontraram nela uma boa opção de comércio devido sua aceitação pelos consumidores.

\subsection{Descrição das espécies}

Para a maioria dos pescadores (95\%), todos os peixes são diferenciados entre peixes adultos e filhotes pelo seu tamanho, ou seja, os adultos são maiores que os filhotes. A diferença entre os peixes machos e fêmeas é através da ova do peixe, pois segundo eles não existe outra forma de se distinguir. Resultado semelhante foi visto por Massena et al., (2014), os pescadores de Vila Cachoeira, Bahia, identificam os peixes da mesma forma que foi relatado no presente trabalho.

A grande maioria dos peixes não apresenta caracteres sexuais secundários, sendo necessário dissecar os indivíduos para a observação das gônadas e identificação do sexo (Vazzoler, 1996).

\subsection{Etnoconhecimento sobre a distribuição espacial dos peixes}

A interação entre diferentes espécies de peixes em um ambiente que fornece variações ao longo da sua extensão faz com que cada grupo de indivíduos busque locais específicos, no qual encontram as melhores condições para sua sobrevivência (Silva et al., 2021). Através das entrevistas, pode-se verificar que os pescadores diferenciam os ambientes de acordo com os locais onde estes são capturados e as formas utilizadas para captura, expressando com isso a posição que os peixes ocupam na 
coluna d'água. Dessa forma, notam-se dois tipos de distribuição: horizontal e vertical.

Neste trabalho, foi analisada apenas a relação por distribuição vertical. Os peixes foram classificados pelos pescadores em "peixes de superfície", "peixes de meia água" e "peixes de fundo" (Tabela 2).

Tabela 2. Conhecimento dos pescadores sobre a distribuição vertical das etnoespécies.

\begin{tabular}{lccc}
\hline & & Distribuição vertical & \\
\hline Peixe & Superfície & Meia água & Fundo \\
\hline Cabeça seca & & & $\mathrm{X}$ \\
Cangati & $\mathrm{X}$ & & \\
Cará bicudo & $\mathrm{X}$ & $\mathrm{X}$ & \\
Cará roxo & $\mathrm{X}$ & $\mathrm{X}$ & $\mathrm{X}$ \\
Cascudo & & & \\
Corvina & & $\mathrm{X}$ & \\
Pataca & $\mathrm{X}$ & $\mathrm{X}$ & \\
Piaba & $\mathrm{X}$ & & $\mathrm{X}$ \\
Piau & $\mathrm{X}$ & $\mathrm{X}$ & $\mathrm{X}$ \\
Piranha branca & $\mathrm{X}$ & $\mathrm{X}$ & \\
Sarapó & & & $\mathrm{X}$ \\
Tambaqui & & $\mathrm{X}$ & \\
Tilápia & $\mathrm{X}$ & $\mathrm{X}$ & \\
Traíra & & & \\
\hline
\end{tabular}

Fontes: Autores.

Esse conhecimento dos pescadores sobre a ocorrência no ambiente e a distribuição espacial dos peixes, construída pela observação ou transmissão oral pode ser confirmado na literatura. As descrições são similares para as espécies do gênero Leporinus (piau), quanto a ocorrência deste peixe tanto em partes fundas do leito, quanto nas margens, em meio a vegetação, além de também ser capturado em trechos com correntezas e/ou com fundo rochoso (Vieira \& Birindelli, 2005).

A época e as situações em que se encontram as espécies, podem também influenciar na posição de cada espécie (Ramires et al., 2006). Como por exemplo, durante o período reprodutivo as espécies podem estar temporariamente no fundo para o desenvolvimento do seu processo reprodutivo, assim como podem oscilar entre a meia água e a superfície para se alimentarem.

\subsection{Reprodução}

Os pescadores associam a época de reprodução com o comportamento de desova, ou seja, nos períodos em que são observados os peixes "ovados", portanto estão se reproduzindo. Foram determinados padrões reprodutivos para 13 espécies (Figura 1). Alguns pescadores foram mais específicos quanto à época de reprodução, pois citaram os meses em que os peixes estão se reproduzindo. Algumas opiniões mostraram-se diversificadas em relação aos meses exatos em que se reproduzem os peixes. 
Figura 1. Períodos de reprodução das espécies de peixes segundo o conhecimento dos pescadores.
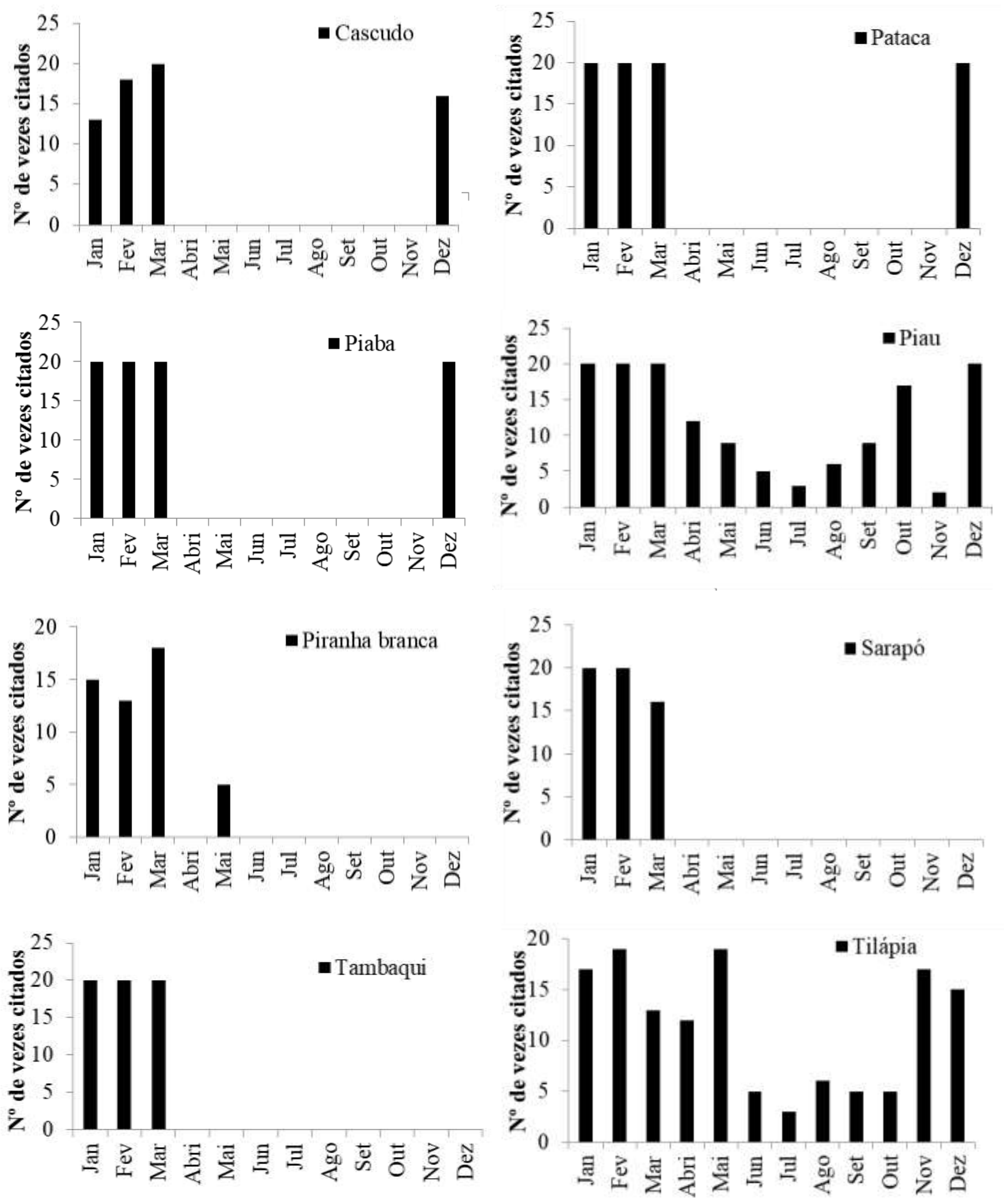


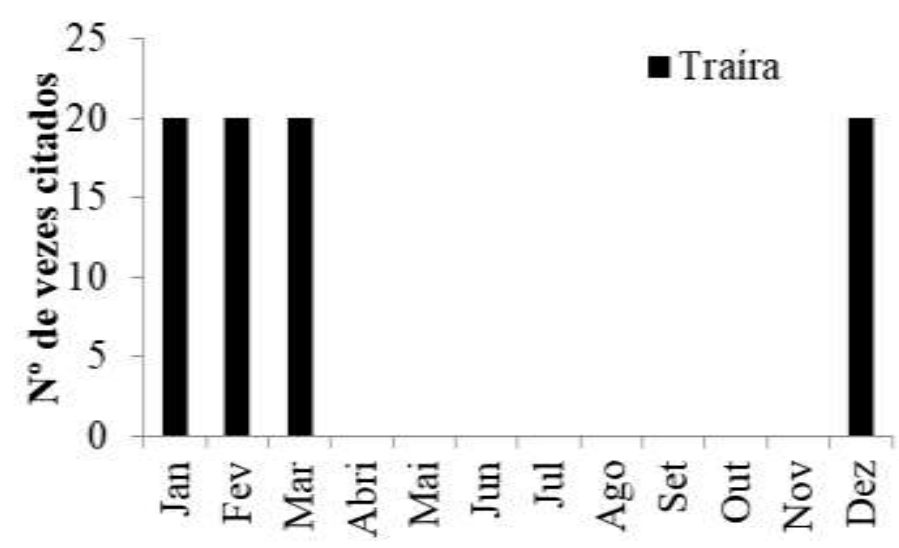

Fontes: Autores.

No caso do sarapó (Gymnotus carapo), tambaqui (Colossoma macropomum) e cabeça-seca (Hoplerythrinus unitaeniatus), todos os pescadores concordaram que entre os meses de janeiro a março essas espécies estão se reproduzindo.

Um estudo realizado por Silvano e Begossi (2002) com pescadores do Rio Piracicaba, São Paulo, relataram que dentre os vários aspectos de etnoecologia (habitat, alimentação, sazonalidade e reprodução), os pescadores apresentaram mais dúvidas em relação à reprodução dos peixes que em relação aos demais aspectos. Os pescadores demonstram um conhecimento mais detalhado em relação a espécies de peixes mais abundantes e mais úteis, especialmente as mais valiosas comercialmente.

\subsection{Status de conservação}

Das 14 etnoespécies relatadas, 32\% dos pescadores relataram que há um desaparecimento dessas espécies e $22 \%$ afirmam que houve uma diminuição da quantidade capturada. Resultado semelhante foi encontrado por Fuzetti e Corrêa (2009), em um estudo na Ilha do Mel, onde os pescadores também relataram a diminuição nos estoques pesqueiros.

Dentre as espécies que eles costumavam pescar a 10 anos atrás e não pescam mais ou dificilmente pescam atualmente se destacam a piranha branca (Serrasalmus rhombeus) e o piau (Leporinus cf. friderici) (Figura 2).

Figura 2. Etnoespécies ausentes ou com estoques diminuídos.

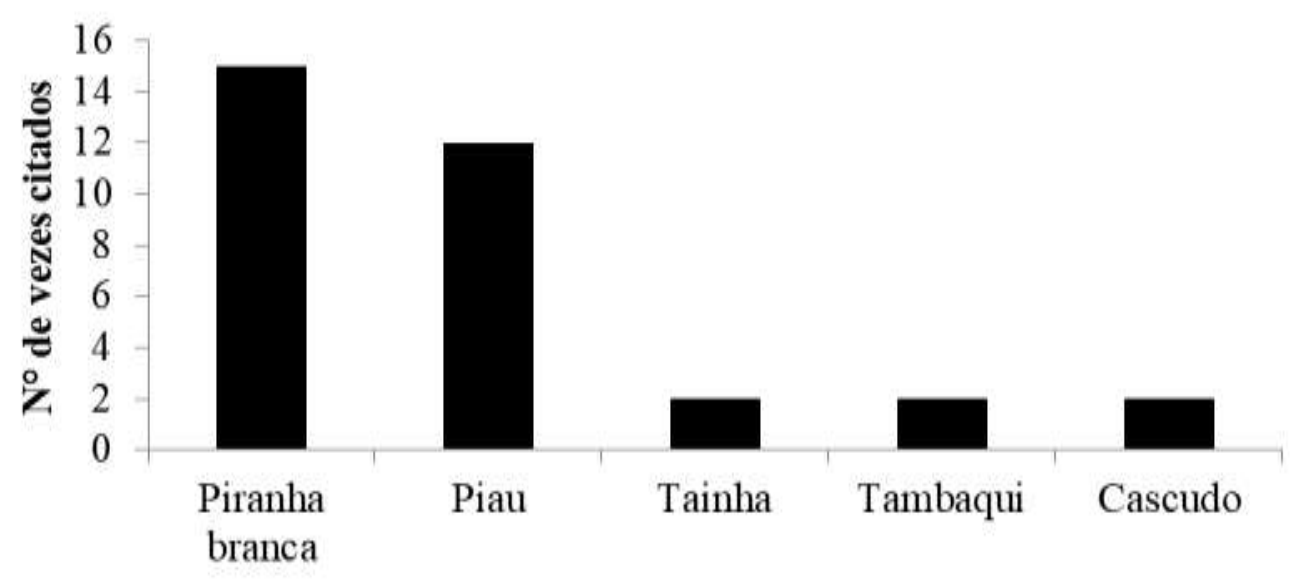

Fontes: Autores.

Quando questionados sobre os principais motivos que ocasionaram a redução das espécies no lago, a maioria (95\%) 
atribuiu ao fato da vegetação nas margens do lago ter diminuído e ao som que as embarcações de turismo fazem ao navegar por essas águas.

Os pescadores de Bebedouro desconhecem o período defeso, afirmam não receber o seguro defeso, dessa forma, não existe um período em que não há pesca.

Essas informações são uteis para o desenvolvimento de estratégias e planos de governo nos aspectos ambientais e sociais, visto que é necessário um plano de manejo para as espécies capturadas devido ao intenso esforço de pesca (Carvalho et al., 2021).

\section{Conclusão}

O conhecimento dos pescadores e a literatura científica observada nesta pesquisa evidencia a crescente importância da etnoictiologia. Os estudos do comportamento do homem e sua relação com o ambiente, através da utilização dos recursos naturais, apontam que resultados provenientes deste tipo de estudo podem ser aplicados na construção de um plano de gestão, em políticas de manejo e investir em educação ambiental.

Os pescadores adquiriram conhecimento das espécies, tal conhecimento envolve os hábitos alimentares, locais de captura e período reprodutivo. Mas com o avanço do turismo e a pouca vegetação, os pescadores notaram que houve uma diminuição no estoque das espécies capturadas.

Na comunidade de Bebedouro, Santo Amaro - MA, a pesca é essencial para a comunidade, uma vez que representa a principal fonte de renda e alimento para os moradores, sendo assim, podemos caracterizar o etnoconhecimento como uma importante ferramenta, considerando a falta de estudo e assistência para o efetivo desenvolvimento de suas atividades, restando apenas os conhecimentos adquiridos através das gerações e que os auxiliam na pesca e conservação dos recursos explorados.

\section{Referências}

Brito, T. P., Oliveira, A. N. D., Silva, D. A. C., \& Rocha, J. A. S. Conhecimento ecológico e captura incidental de tartarugas marinhas em São João de Pirabas, Pará, Brasil. Biotemas, 28(3): 159-175, 2015.

Carvalho, T. C. C., Barros, M. R. F., Ramos, A. J., Reis, A. R., Melo, A. A. D., Palheta, S. C. M. G., Carvalho, A. S. S., Palheta, G. D. A. Socioeconomia e etnoconhecimento de pescadores artesanais da comunidade do Cajueiro, distrito de Mosqueiro, Amazônia Oriental. Research, Society and Development, 10.

Begossi. A. H. N., \& Silvano, A. M. Ecologia Humana, Etnoecologia e Conservação. IN_Amorozo, M. C. M.; Ming; L. C.; Silva; S. P. Métodos de Coleta e Análise de Dados em Etnobiologia, Etnoecologia e Disciplinas Correlatas. UNESP/SBEE/CNPq, Rio Claro, 204 p. 2002.

Da Cunha, F. C. Etnoconhecimento de pescadores no sistema Lago Grande de Manacapuru /. UFAM, 2011.

Diegues, A. C., Arruda, S. V. Silva, V. C. F., Figols, F. A. B., \& Andrade, D. Saberes tradicionais e Biodiversidade no Brasil. Brasilia: Ministério do Meio Ambiente, São Paulo USP, 176p. 2000.

FAO. Food and Agriculture Organization of the United Nations. The state of world fisheries and aquaculture contributing to food security and nutrition for all. Rome. 2016.

Fuzetti, L., \& Corrêa, M. F. M. Perfil e renda dos pescadores artesanais e das vilas da Ilha do Mel - Paraná, Brasil. Boletim do Instituto de Pesca, São Paulo, 35(4): 609-621, 2009.

IBAMA. Instituto Brasileiro do Meio Ambiente e dos Recursos Naturais Renováveis. Plano de Manejo do Parque Nacional dos Lençóis Maranhenses. CD ROOM. 2004.

Ludke, M., \& André, M. E. D. A. Pesquisa em educação: Abordagens qualitativas. EPU, 99p, 1986.

Massena, F. S., Ramos, F. L., Mirotti, P. I., Trevizan, S. D. P., \& Wibelinger, L. M. Etnoictiologia dos pescadores Artesanais da vila Cachoeira, Ilhéus - BA. Revista Brasileira de Engenharia de Pesca, 7(n): 32-44, 2014.

Monteles, J. S., De Almeida Funo, I. C., \& De Castro, A. C. L. Caracterização da pesca artesanal nos municípios de Humberto de Campos e Primeira CruzMaranhão. Boletim do Laboratório de Hidrobiologia, 23.

Piorski, N. M., Ferreira, B. R. A., Guimarães, E. C., Ottoni, F. P., Nunes, J. L. S., \& Brito, P. S. Peixes do Parque Nacional dos Lençóis Maranhenses. São Luís: Café \& Lápis; Edufma, 189 p. 2017. 
Research, Society and Development, v. 10, n. 8, e52510817545, 2021

(CC BY 4.0) | ISSN 2525-3409 | DOI: http://dx.doi.org/10.33448/rsd-v10i8.17545

Ramires, M., \& Molina, S. M. G. Influências da Pesca Esportiva no Modo de Vida dos Pescadores Caiçaras do Vale do Ribeira. In: Encontro Latino Americano de Pós-Graduação, 4, 2004, São José dos Campos. Anais [...] São José dos Campos: Universidade do Vale do Paraíba, 2004.

Ramires, M. Molina, S. M. G., \& Hanazaki, N. Etnoecologia caiçara: o conhecimento dos pescadores artesanais sobre aspectos ecológicos da pesca. Revista Biotemas. São Paulo. 108 p. 2006.

Santos, M. P. N., Seixas, S., Aggio, R., Hanazaki, N., Costa, M. F., Schiavetti, A., Dias, J., \& Azeiteiro, U. M. A pesca artesanal enquanto atividade humana: pesca artesanal e sustentabilidade. Revista de gestão costeira integrada, 12(4): 405-427, 2012.

Silva, T. A., Oliveira, W. D. S., \& Sampaio, F. A. C. Etnoconhecimento de pescadores artesanais sobre a ictiofauna do rio Jiquiriçá, Bahia. Ethnoscientia, 6(1).

Silvano, R. A. M., \& Begossi, A. Ethnoichthyology and fish conservation in the Piracicaba river (Brazil). Journal of Ethnobiology, 22 (2): 285-306, 2002.

Vazzoler, A. E. A. de M. Manual de métodos para estudos biológicos de populações de peixes; reprodução e crescimento. CNPq. 1981.

Vieira, F., \& Birindelli, J. L. Leporinus thayeri Borodin, 1929. In: Rosa, R.; \& Lima, F. Livro vermelho da fauna brasileira ameaçada de extinção: peixes. ICMBIO, 2005 